PHILIP PAYTON 



\title{
Philip Payton
}

THE FATHER OF BLACK HARLEM

Kevin McGruder

\author{
必 \\ Columbia University Press \\ New York
}


Columbia University Press

Publishers Since 1893

New York Chichester, West Sussex

cup.columbia.edu

Copyright $\odot 2021$ Columbia University Press

All rights reserved

Library of Congress Cataloging-in-Publication Data

Names: McGruder, Kevin, 1957- author.

Title: Philip Payton : the father of black Harlem / Kevin McGruder.

Description: New York : Columbia University Press, [2021] |

Includes bibliographical references and index.

Identifiers: LCCN 2021002789 (print) | LCCN 2021002790 (ebook) | ISBN 9780231198929

(hardback) | ISBN 9780231198936 (trade paperback) | ISBN 9780231552875 (ebook)

Subjects: LCSH: Payton, Philip, 1876-1917. | Real estate developers-New York (State)—

New York-Biography. | African American businesspeople-New York (State)—New York-

Biography. | Real estate development-New York (State) -New York-History-2oth century. |

African Americans-Housing-New York (State)—New York-History-2oth century. |

Discrimination in housing-New York (State)-New York-History-2oth century. | Harlem

(New York, N.Y.)--History. | Harlem (New York, N.Y.)—Biography.

Classification: LCC HD268.N5 M34 2021 (print) | LCC HD268.N5 (ebook) | DDC 333.33092 $[\mathrm{B}]-\mathrm{dc} 23$

LC record available at https://lccn.loc.gov/2021002789

LC ebook record available at https://lccn.loc.gov/202100279o

Columbia University Press books are printed on permanent and durable acid-free paper.

Printed in the United States of America

Cover design: Julia Kushnirsky

Cover photographs: Photographs and Prints Division, Schomburg Center for Research in Black Culture, New York Public Library 
To the memory of

Dr. Judith Stein, advisor, mentor, friend and

Dr. Martia Graham Goodson, friend and mentor 
